\title{
Prescribing Patterns of Antidepressants among Depressive Men in Malaysia: A Survey
}

\author{
Abdul Rahman Fata Nahas ${ }^{1,2^{*}}$, Syed Azhar Syed Sulaiman ${ }^{1}$ \\ 'Discipline of Clinical Pharmacy, School of Pharmaceutical Sciences, Universiti Sains Malaysia, Penang, MALAYSIA. \\ 2Pharmacy Practice Department, Kulliyyah of Pharmacy, International Islamic University Malaysia, Pahang, MALAYSIA.
}

\begin{abstract}
Objective: In recent studies on the prescribing pattern of antidepressants, selective serotonin reuptake inhibitors (SSRIs) were the most prescribed antidepressants in many countries. Yet, little reports investigated depressive populations for antidepressants prescribing in Malaysia. Therefore, this study aimed to explore the pattern of prescribed antidepressants in a Malaysian group of depressive patients throughout their years of depression. Method: A cross-sectional study was conducted on 119 adult male depressive outpatients who were recruited from three psychiatric centers. Patients' age and duration of depression were recorded, as well as their current and past antidepressants prescribed. Results: Of the study participants, 107 patients (89.9\%) were currently antidepressant-treated. Mean age of patients was $49.9(S D=11.0)$, while mean duration of depression was 7.8 years $(S D=6.1)$. Markedly, SSRIs were the most currently prescribed antidepressants $(72.9 \%)$, followed by tricyclic antidepressants (TCAs; $10.3 \%)$. Moreover, SSRIs predominated the prescribed antidepressants over the years of depression. Additionally, there was an increase in the share of SSRIs prescribing during these years in the expenses of other antidepressants, especially TCAs, whose prescription share fell from $34.1 \%$, in the years preceding 2005 , to only $10.3 \%$ in the current time. Conclusion: In sum, this study findings concur several reports from various countries, in which SSRI were the most frequent prescribed antidepressants and their prescribing was increasing over nearly the last
\end{abstract}

10 years. Further research is advocated to explore these findings in larger depression populations.

Key words: Antidepressants, Depression, Malaysia, Men, Prescribing pattern

\section{Key message}

There is little published research concerning the prescribing pattern of antidepressants in Malaysia.

The pattern of antidepressants prescribing was investigated in 107 depressive men.

Selective serotonin reuptake inhibitors predominated the prescribed antidepressants throughout the years of diagnosed depression.

Further studies in larger population are warranted to confirm these findings.

\section{Correspondence:}

Abdul Rahman Fata Nahas, Pharmacy Practice Department, Kulliyyah of Pharmacy, International Islamic University Malasia (IIUM), Jalan Sultan Haj ahmad Shah, Kuantan, 25200, Pahang, MALAYSIA.

Phone: +601 33726 073, Fax: +604 6570017

Email: abd_mfn@hotmail.com

DOI: 10.5530/jyp.2018.10.22

\section{INTRODUCTION}

Depression is a common mental disorder characterized by a low mood status. People with depression might show significant impaired physical and mental features. ${ }^{1,2,3}$ According to the World Health Organization, ${ }^{4}$ the lifetime prevalence of depression is $17 \%$, with over 350 million around the world being affected. Depression also is ranked globally as the $11^{\text {th }}$ greatest cause of disability and mortality..$^{5}$ Although people of all ages from both sexes are potentials for depression, depression among men is less prevalent than women. ${ }^{6}$ Similar to all world populations, depression is alarming among Asians, yet it is less prevailing. ${ }^{7,8}$ In Malaysia, depression is one of the most important disorders, with almost 2.3 million Malaysians are expected to be affected at a certain point in their lives. ${ }^{9}$

With the fact that depression is a highly devastating disorder, it is crucial for healthcare providers to address their depressive patients appropriately, and to provide a suitable management for them. Clinically, depression is mainly mitigated non-pharmacologically through, for instance, psychotherapy, as well as pharmacologically through principally antidepressants. In the 1950s, the fortuitous discovery of the mood elevating effect of the monoamine oxidase inhibitors (MAOIs) open the door for a new era in the management of depression. ${ }^{10}$ By the end of the same decade, iproniazid, a MAOI, and imipramine, a tricyclic antidepressant (TCA), were introduced to the clinical community. Following this step, another revolutionary step was achieved in the 1980s, with the introduction of fluoxetine, the first member of the totally new antidepressants' family; i.e., the selective serotonin reuptake inhibitors (SSRIs). ${ }^{11}$ Starting from the early 2000s, an increment in not only the numbers, but also the types of antidepressants was at hand for clinical practice, ${ }^{12}$ which provides several choices for clinicians for a better and safer selection of medications for the management of depressive disorders.

TCAs and tetracyclic antidepressants (TeCAs) were the first-line drugs for depression treatment over several years. Recent drug utilization studies; however, have demonstrated that SSRIs are the preferable antidepressants in many countries, as apparent from several studies across various populations. ${ }^{8,12,13,14,15,16}$ Even so, SSRIs hold no superiority in terms of efficacy when compared to other antidepressants. ${ }^{12}$

There is little published research concerning the prescribing pattern of antidepressants in Malaysia. The Research on Asia Psychotropic Prescription Pattern (REAP) project aimed to investigate the prescribing pattern of antidepressants among several Asian populations, including Malaysia. ${ }^{8}$ Although the REAP project was not focusing specifically on depressive patients, a published paper by Chen and colleagues in 2015 presented the findings from analyzing some of the data of the REAP project pertinent to depressive patients. ${ }^{17}$ Another paper was published from the same project by Chee and associates in 2015 highlighted some 
of the findings from the REAP project, but not especially among depressive patients. ${ }^{16}$ In our study, we aimed to determine the pattern of current prescribed antidepressants in a Malaysian depression population, we also traced the prescription patterns of antidepressants among our patients over the period of their diagnosed depression.

\section{METHODS}

\section{Study sample}

This study was part of a 10 months, cross-sectional study among depressive men in Malaysia that was started in May 2015. The sample included 119 outpatients from three psychiatric departments in three general hospitals. Adult male patients were recruited if they had been diagnosed with major depressive disorder (MDD) and could understand the study aim. The study concept and aim were clearly explained to the participants, and those who agreed provided written informed consent. Prior to the study implementation, the study was revised and approved by the Medical Research and Ethics Committee, Ministry of Health, Malaysia.

\section{Criteria of depression}

Diagnosis of depression was confirmed by the departments' psychiatrists according to the Malaysian's Guidelines in MDD management, ${ }^{18}$ which adopt the endorsed depression diagnostic criteria of the fourth version of the Diagnostic and Statistical Manual of Mental Disorders (DSM-IV) or the International Classification of Disease, Tenth Edition (ICD-10).

\section{Antidepressants classification}

Based on their pharmacological classification, ${ }^{19}$ antidepressants were classified into six classes, including TCAs, TeCAs, SSRIs, serotoninnorepinephrine reuptake inhibitors (SNRIs), MAOIs and other antidepressants. Other antidepressants represented all other antidepressants not falling in the classes mentioned.

\section{Data collected}

Relevant to the present study, we recorded participants' age, period of depression since its diagnosis (depression duration), current use of antidepressants, as well as the history of prescribed antidepressants over the depression duration of our subjects.

\section{Statistical analyses}

The data were analyzed utilizing the Statistical Package for Social Sciences version 22.0 (SPSS Inc., Chicago, IL, USA). Means and standard deviations $(S D)$, or frequencies and percentages, wherever appropriate, were presented to provide the description of the study data. We also categorized antidepressants prescribing period into four different groups; current (2015), between 2014-2010, between 2009-2005, and before 2005.

\section{RESULTS}

Of the recruited subjects, 107 patients (89.9\%) were currently on antidepressant medications, while 12 patients were not antidepressant-treated. The sample age range was 24 to 73 years, with a mean of $49.9(S D=11.0)$. Mean depression duration was 7.8 years $(S D=6.1)$, ranging from .3 to 25 years.

\section{Current antidepressants prescribed}

The different classes of antidepressants that were currently prescribed are presented in Table 1. SSRIs comprised the most frequent antidepressants currently being consumed (72.9\%), followed by TCAs, whereas MAOIs were the least frequent antidepressants.

\section{Classes of antidepressants prescribed over the period of depression}

In addition to reporting the current prescribed antidepressants, antidepressants consumed by the patients in the last five years, between 2005

\begin{tabular}{|c|c|}
\hline Antidepressants Class & $\mathrm{n}, \%$ \\
\hline SSRIs & $78(72.9 \%)$ \\
\hline TCAs & $11(10.3 \%)$ \\
\hline SNRIs & $9(8.4 \%)$ \\
\hline TeCAs & $6(5.6 \%)$ \\
\hline MAOIs & $3(2.8 \%)$ \\
\hline
\end{tabular}

Note. SSRIs, Selective serotonin reuptake inhibitors; TCAs, Tricyclic antidepressants; SNRIs, Serotonin-norepinephrine reuptake inhibitors; TeCAs, Tetracyclic antidepressants; MAOIs, Monoamine oxidase inhibitors.

and 2009, and before the year 2005 were reported. The increase in antidepressants prescribing is nearly entirely in favor of the SSRIs. Although there has been a small increase in the prescribing of SNRIs, this is insignificant in comparison to the increase in the SSRIs prescribing. In the years preceding 2005, TCAs and SSRIs represented $34.1 \%$ and $45.5 \%$ of all antidepressants prescribed; respectively. However, results showed that there was a significant shift in the current prescribing pattern towards SSRIs prescribing, which had raised up to $72.9 \%$ of all antidepressants Figure 1.

\section{DISCUSSION}

To the best of our knowledge, current study is the first among specifically depressive men in Malaysia that surveyed current and past prescribing patterns of antidepressants for a depressive group of patients. Although our findings represented a small number of patients, it highlighted that antidepressants prescribing was currently in favor of SSRIs, whose prescribing increased during the years studied. These findings were in parallel with several studies conducted throughout the world.

In 2004, Gunnell and Ashby reported that SSRIs, in the expense of other antidepressants, were the most prescribed antidepressants in the United Kingdom. ${ }^{13}$ In another nation-based study in New Zealand, ${ }^{14}$ SSRIs prescribing increased from $41 \%$ in 1995 to $57 \%$ in 2005 , while the TCAs prescribing declined from $59 \%$ to $38 \%$ during the same period. Although these findings were not solely related to the antidepressants prescribed for depressive disorders, yet the greater percentage of antidepressants prescribed for depressive disorders was of SSRIs; i.e. $64 \%$. Similar findings were documented by Bauer and colleagues in $2008,{ }^{12}$ who studied the prescribing patterns of antidepressants in a population-based, multinational study across Europe. More than half of the depressive patients (63.3\%) recruited were prescribed SSRIs. These findings were boosted by Olfson and Marcus study in 2009, ${ }^{15}$ which revealed that from 1996 to 2005 , SSRIs prescribing in the US was increased from $54.8 \%$ to $66.9 \%$, whereas the TCAs prescribing declined from $35 \%$ to $11.1 \%$. Recently, Malaysia was represented by 161 Malaysian psychiatrists in the REAP project, a physician-based multi-national survey in 2013, which encompassed 10 Asian countries. The project showed that SSRIs were the most common antidepressants clinically prescribed, not only for depression, but also for patients with other mood disorders or somatoform disorders. ${ }^{8,16,17}$ In fact, what defines the decision for the selection of a particular antidepressant or a specific class is determined by several factors, some are related to the treatment and others are related to the physician or the patients themselves. For instance, the previous use of an antidepressant, or a combination of antidepressants may influence the decision of antidepressant choice. Moreover, physician's age, gender and specialty in 


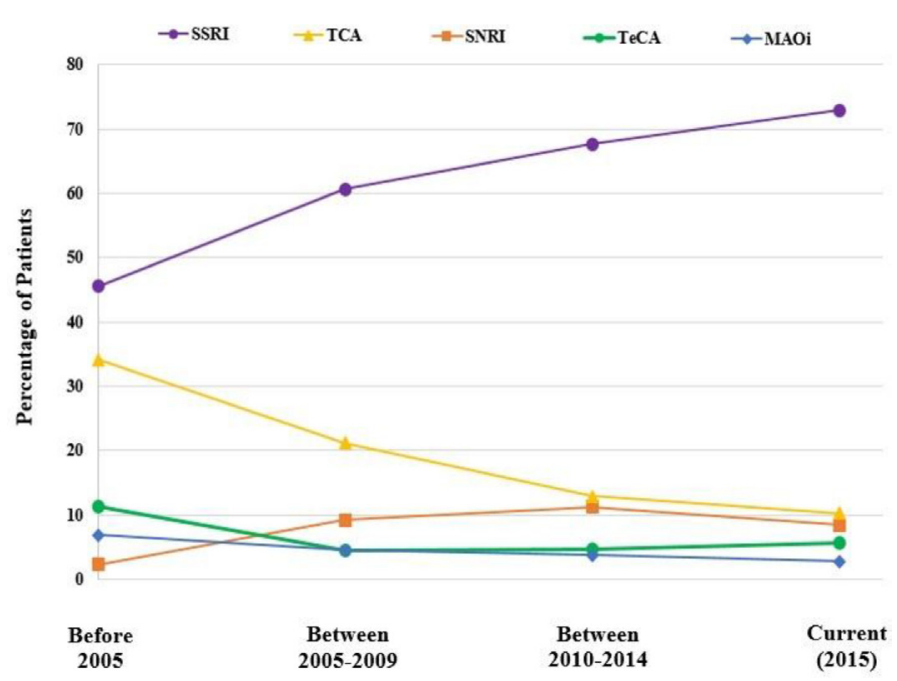

Figure 1 : Percentages of antidepressant medications prescribed, by drug class, over the years of diagnosed depression of the study subjects. SSRIs, Selective serotonin reuptake inhibitors; TCAs, Tricyclic antidepressants; SNRIs, Serotonin-norepinephrine reuptake inhibitors; TeCAs, Tetracyclic antidepressants; MAOIs, Monoamine oxidase inhibitors.

addition to the severity of depression, patient's age or comorbidities, all can affect the preferred antidepressant upon therapy initiating. ${ }^{12}$

On one hand, all antidepressants, including SSRIs, have an increased risk for suicide in younger adults aged less than 25 years. ${ }^{20}$ On the other hand, SSRIs are believed to be as effective as other classes of antidepressants. ${ }^{12,14}$ There is also no empirical evidence to support a recommendation for a specific second generation antidepressants based on efficacy. ${ }^{20}$ Even so, the previous discussion showed that the global prescribing pattern in the last few years and hitherto is in favor of SSRIs prescribing. Therefore, the possible factors suggesting for this paradigm shift are discussed in the next paragraph.

First, SSRIs have advantageous features related to the dosing. This can be seen in their once daily dosing and the need for less dose titration compared to TCAs. ${ }^{12}$ Second, when considering for prescribing a medication, the effectiveness is not the only one that is looked for; the safety is a critical issue, and this applicable to the selection of SSRIs, which shows fewer side effects compared to TCAs, ${ }^{12}$ they are also much more tolerated compared to other antidepressants. ${ }^{21}$ Furthermore, SSRIs results in less overdose-related toxicities, in turn, the increase in SSRIs prescribing might limit the access to other antidepressants that are more toxic. ${ }^{13}$ Lastly, the national guidelines may play a key role in the selection of antidepressants. ${ }^{12}$ This might have paved the way for SSRIs to predominate other antidepressants in the clinical settings. Relevant to the latter point, the Malaysian's guidelines in the management of MDD recommend SSRIs as the first line antidepressants, for both the mild MDD and the moderate to severe MDD. On top of that, if switching to another antidepressant agent is prompted, the guidelines still recommend considering other SSRIs, although an agent from a different class is also recommended. ${ }^{18}$

This study has several limitations. First is the small size of the sample studied, which would not allow for generalizing our findings. Next, factors influencing the patterns of antidepressants prescribing were not explored. Moreover, our cohort was selected from hospitals, and no inclusion of a sample from rural area and small primary clinical settings was done, which can also affect generalizability of the findings.

\section{CONCLUSION}

All in all, our data suggest that SSRIs are more likely to be prescribed for depressive men in a clinical setting in Malaysia, which is in parallel with various studies conducted in many countries. SSRIs were also the most prescribed over almost the last ten years. This seems to be in line with the Malaysian's guidelines in MDD management; however, clinicians should keep in mind that all antidepressants had an increased risk for suicidality in younger patients, and that SSRIs are not superior in efficacy compared to other antidepressants. Further studies are recommended to investigate the study findings while addressing the limitations raised.

\section{ACKNOWLEDGMENT}

Many thanks to Dr. Ramli Musa (International Islamic University Malaysia), Dr. Ahmad Zafri, Dr. Nor Hayati Arif and Dr. Salina Abdul Aziz (Ministry of Health, Malaysia) for their assistance in patients recruitment.

\section{CONFLICTS OF INTEREST}

Abdul Rahman Fata Nahas and Syed Azhar Syed Sulaiman declare that they have no conflict of interest.

\section{FUNDING INFORMATION}

This work was funded in part by Research Initiative Grant Scheme (RIGS 17-005-0580) from International Islamic University Malaysia (IIUM).

\section{REFERENCES}

1. Hichiya $H$, Mimura $Y$, Hanioka N. Evaluation of oral antidepressant drugs for adaptation to the simple suspension method. J Young Pharm. 2014;6(4):56-60.

2. Nelson JC, Charney DS. The symptoms of major depressive illness. Am J Psychiatry. 1981;138(1):1-13.

3. The ICD-10 Classification of Mental and Behavioural Disorders: Diagnostic criteria for research [Internet]. World Health Organization; 1993 [cited 2017 June 14]. Available from: http://www.who.int/classifications/icd/en/GRNBOOK.pdf.

4. Depression [Internet]. World Health Organization. [updated 2017 Feb 1; cited 2017 May 4]. Available from: http://www.who.int/mediacentre/factsheets/fs369/en/.

5. Murray CJ, Vos T, Lozano R, Naghavi M, Flaxman AD, Michaud $C$, et al. Disability-adjusted life years (DALYs) for 291 diseases and injuries in 21 regions, 1990-2010: a systematic analysis for the Global Burden of Disease Study 2010. Lancet. 2012;380(9859):2197-223.

6. Kessler RC. Epidemiology of women and depression. J Affect Disord. 2003;74(1):5-13.

7. Chiu E. Epidemiology of depression in the Asia Pacific region. Australas Psychiatry. 2004;12(Sup 1):s4-s10.

8. Naotaka S. Research on Asian prescription patterns (REAP): focusing on data from Japan. Taiwanese Journal of Psychiatry. 2014;28(2):72-95

9. Mukhtar F, Oei TP. A review on assessment and treatment for depression in Malaysia. Depress Res Treat. 2011;2011:1-8

10. Ferguson JM. SSRI antidepressant medications: adverse effects and tolerability. Prim Care Companion. J Clin Psychiatry. 2001;3(1):122-7.

11. López-Muñoz F, Alamo C. Monoaminergic neurotransmission: the history of the discovery of antidepressants from 1950s until today. Curr Pharm Des. 2009;15(14):1563-86.

12. Bauer M, Monz BU, Montejo AL, Quail D, Dantchev N, Demyttenaere K, et al. Prescribing patterns of antidepressants in Europe: results from the Factors Influencing Depression Endpoints Research (FINDER) study. Eur Psychiatry. 2008;23(1):66-73.

13. Gunnell D, Ashby D. Antidepressants and suicide: what is the balance of benefit and harm. BMJ. 2004;329(7456):34-8.

14. New Zealand Ministry of Health. Patterns of Antidepressant Drug Prescribing and Intentional Selfharm Outcomes in New Zealand: An ecological study, 2007 [Internet]. Wellington (New Zealand): Ministry of Health; 2007. [cited 2016 Aug 16]. Available from: http://www.health.govt.nz/publication/patterns-antidepressantdrug-prescribing-and-intentional-self-harm-outcomes-new-zealand-ecological.

15. Olfson M, Marcus SC. National patterns in antidepressant medication treatment. Arch Gen Psychiatry. 2009;66(8):848-56.

16. Chee KY, Tripathi A, Avasthi A, Chong MY, Sim K, Yang SY, et al. International study on antidepressant prescription pattern at 40 major psychiatric institutions and hospitals in Asia: A 10-year comparison study. Asia Pac Psychiatry. 2015;7(4):366-74 
17. Chen C, Si TM, Xiang YT, Ungvari GS, Wang CY, He YL, et al. Prevalence and prescription of antidepressants in depression with somatic comorbidity in Asia: the research on East Asian psychotropic prescription patterns study. Chin Med J. 2015;128(7):853-8.

18. Malaysia Ministry of Health. Clinical Practice Guidelines, Management of Major Depressive Disorder, 2007 [Internet]. Malaysia: Ministry of Health; 2007. [cited 2016 Jan 23]. Available from: http://www.acadmed.org.my/view_file. cfm?fileid=250

19. Linde K, Kriston L, Rücker G, Jamil S, Schumann I, Meissner K, et al. Efficacy and acceptability of pharmacological treatments for depressive disorders in primary care: systematic review and network meta-analysis. Ann Fam Med. 2015;13(1):69-79.

20. Gartlehner G, Hansen RA, Morgan LC, Thaler K, Lux L, Noord VM, et al. Comparative Benefits and Harms of Second-Generation Antidepressants for Treating Major Depressive DisorderAn Updated Meta-analysis. Ann Intern Med. 2011;155(11):772-85

21. National Institute for Clinical Excellence. Depression in adults: recognition and management, 2009 [Internet]. NICE Clinical guideline CG90; 2009. [cited 2016 Nov 19]. Available from: https://www.nice.org.uk/guidance/cg90.

Article History: Submission Date : 23-07-2017 ; Revised Date : 29-08-2017; Acceptance Date : 29-09-2017.

Cite this article: Nahas ARF, Sulaiman SAS. Prescribing Patterns of Antidepressants among Depressive Men in Malaysia: A Survey. J Young Pharm. 2018;10(1):98-101. 